\title{
Phonon engineering with superlattices: Generalized nanomechanical potentials
}

\author{
O. Ortíz, M. Esmann, and N. D. Lanzillotti-Kimura $\odot^{*}$ \\ Centre de Nanosciences et de Nanotechnologies (C2N), CNRS, Université Paris-Sud, Université Paris-Saclay, \\ 10 Boulevard Thomas Gobert, 91120 Palaiseau, France
}

(Received 11 February 2019; published 22 August 2019)

\begin{abstract}
Phonons are a promising simulation platform for single particles trapped in quantum wells, interatomic molecular dynamics, and, in general, potentials. Earlier implementations to simulate coherent wave propagation in one-dimensional potentials using acoustic phonons with gigahertz-terahertz frequencies were based on coupled nanoacoustic resonators. Here we generalize the concept of adiabatic tuning of periodic superlattices for the implementation of effective one-dimensional potentials giving access to cases that cannot be realized by previously reported phonon engineering approaches, in particular the acoustic simulation of electrons and holes in a quantum well or a double-well potential. In addition, the resulting structures are much more compact and hence experimentally feasible. We demonstrate that potential landscapes can be tailored with great versatility in these multilayered devices, apply this general method to the cases of parabolic, Morse, and double-well potentials, and study the resulting stationary phonon modes. The phonon cavities and potentials presented in this work could be probed by all-optical techniques like pump-probe coherent phonon generation and Brillouin scattering.
\end{abstract}

DOI: 10.1103/PhysRevB.100.085430

\section{INTRODUCTION}

Nanophononics addresses the control of acoustic phonons in solid state structures with engineered acoustic impedance modulations [1-5]. Commonly studied nanoacoustic devices include phonon mirrors, filters, and resonant cavities to shape the interaction of phonons at the $\mathrm{GHz}-\mathrm{THz}$ frequency scale with both light and electronic states [4,6,7]. Applications include fast modulators in semiconductor lasers [8], novel approaches for the generation of $\mathrm{THz}$ radiation [9], and the nanomechanical characterization of biological tissue [10-12]. Optical tools such as ultrafast pump-probe spectroscopy and inelastic Brillouin scattering have enabled the study of phononic spectra, temporal dynamics, and coherence properties on the nanoscale [13-20]. This paved the way to establish nanoacoustics also as a platform for the simulation of wave dynamics in electronic systems [21-23]. In contrast to optical platforms, nanophononics features the particular advantage of a slow speed of propagation compared to light and a long coherence length in the range of hundreds of micrometers [20] at wavelengths in the $10 \mathrm{~nm}$ range. Therefore, coherent propagation of phonons can be studied in quasi-infinite systems in which wave dynamics can be optically probed on timescales well below the mechanical oscillation period.

The effect of an electric potential on a charged particle is to accelerate it. In this work we mimic this effect in a phononic

\footnotetext{
*daniel.kimura@c2n.upsaclay.fr
}

Published by the American Physical Society under the terms of the Creative Commons Attribution 4.0 International license. Further distribution of this work must maintain attribution to the author(s) and the published article's title, journal citation, and DOI. system by locally changing the group velocity of longitudinal acoustic phonons in semiconductor multilayers. A wellestablished building block in the engineering of acoustic phonons is the nanoresonator based on the acoustic counterpart of an optical Fabry-Perot cavity [6,20]. Most of the earlier approaches to implement effective potentials with phonons are based on engineering band structures arising from coupled nanoacoustic cavities [22], i.e., the phononic equivalent of the coupled resonator optical waveguides (CROWs) [24]. Such devices have been used to, e.g., mimic wave dynamics in Wannier-Stark ladders showing Bloch oscillations [25] or topological effects in polyacetylene [26]. In contrast to coupled cavities, another approach to study wave dynamics in effective acoustic potentials is to introduce a progressive change in the parameters of the cells along a periodic multilayer structure. Very recently, an acoustic cavity was reported based on the adiabatic periodicity breaking of a superlattice [5], in analogy to a potential well. Exploiting the symmetry properties in periodic superlattices, topological interface modes have also been recently reported [27-29].

Here we generalize the adiabatic tuning of the period thickness in a superlattice for the implementation of effective onedimensional potentials. Up to now, the realization of effective phononic potentials was predominantly based on coupled resonant cavities exploring the tight-binding physics of phonons tunneling between sites on a lattice [22,24-26]. In contrast, here we explore the physics of nearly free electron models based on a single superlattice. We demonstrate that potential landscapes can be tailored with great versatility using significantly thinner structures than previously reported, rendering even the implementation of complicated effective potentials experimentally feasible. We apply this general method to the cases of parabolic, Morse, and double-well potentials and study the resulting stationary phonon modes. 
The paper is organized as follows: Section II presents a general theoretical framework to engineer acoustic cavities based on adiabatic changes in the local acoustic band structures. We present a first case mimicking the confinement of an electron and a hole in a quantum well. In Sec. III the use of these tools is extended for the implementation of effective parabolic, double-well, and Morse potentials and their stationary acoustic modes are calculated. Section IV addresses the role of acoustic absorption losses in these resonators. Conclusions and perspectives of this work are presented in Sec. V.

\section{ACOUSTIC POTENTIAL WELLS}

The theoretical framework to engineer these effective potentials is based on the dispersion relation of phonons in a periodic superlattice [30] with each unit cell composed of two layers. Considering AlAs and GaAs as the materials for these layers, we parametrize this dispersion relation as

$$
\begin{aligned}
\cos (q d)= & \cos \left(\frac{\omega D}{f_{d}}\right) \\
& -\frac{\epsilon^{2}}{2} \sin \left(\frac{\omega r D}{f_{d}}\right) \sin \left(\frac{\omega(1-r) D}{f_{d}}\right),
\end{aligned}
$$

with

$$
\epsilon=\frac{Z_{\mathrm{GaAs}}-Z_{\mathrm{AlAs}}}{\left(Z_{\mathrm{GaAs}} Z_{\mathrm{AlAs}}\right)^{1 / 2}} .
$$

Here $q$ is the phonon quasimomentum, $d=d_{\mathrm{AlAs}}+d_{\mathrm{GaAs}}$ is the geometric unit cell thickness (i.e., structure period) composed of two layers, and $\omega$ is the angular frequency. $Z_{\mathrm{GaAs}}=\rho_{\mathrm{GaAs}} v_{\mathrm{GaAs}}$ and $Z_{\mathrm{AlAs}}=\rho_{\mathrm{AlAs}} v_{\mathrm{AlAs}}$ are the acoustic impedances of the two materials where $\rho_{\text {AlAs }}$ and $v_{\text {AlAs }}$ $\left(\rho_{\mathrm{GaAs}}\right.$ and $\left.v_{\mathrm{GaAs}}\right)$ are the mass density and the speed of sound, respectively. We denote the acoustic path length of a unit cell $D=D_{\mathrm{AlAs}}+D_{\mathrm{GaAs}}$ with the contributions $D_{\mathrm{AlAs}}=$ $d_{\mathrm{AlAs}} f_{d} / v_{\mathrm{AlAs}}$ and $D_{\mathrm{GaAs}}=d_{\mathrm{GaAs}} f_{d} / v_{\mathrm{GaAs}}$ from the two layers, which are measured in units of wavelength at a design frequency $f_{d}$. We furthermore introduced the parameter

$$
r=\frac{D_{\mathrm{AlAs}}}{D}
$$

which describes the relative contribution of the AlAs layer to the overall acoustic path length of the unit cell. The dispersion relation described in Eq. (1) is plotted in the leftmost panel of Fig. 1(a) for a GaAs/AlAs superlattice with $f_{d}=300 \mathrm{GHz}$, $D=1$, and $r=0.25$, i.e., a phonon at the design frequency $f_{d}$ acquires a propagation phase of $2 \pi$ upon traversing one unit cell and a phase of $\pi / 2$ upon traversing one AlAs layer. We observe three acoustic bands separated by two acoustic minigaps around 150 and $300 \mathrm{GHz}$ in which only evanescent phonons are solutions to the wave equation. Since $D$ is chosen as an integer multiple of $1 / 2, f_{d}$ lies at the center of a minigap, in this case the second one [30]. The dashed red rectangle in the second minigap marks the region of the band structure for which zoom-ins are presented in the right part of Fig. 1(a) for different values of the parameter $r$. Importantly, we observe that the spectral width of the minigap directly depends on the value of $r$, i.e., on the relative acoustic thickness of the two layers constituting the unit cell $[27,30]$. Starting from
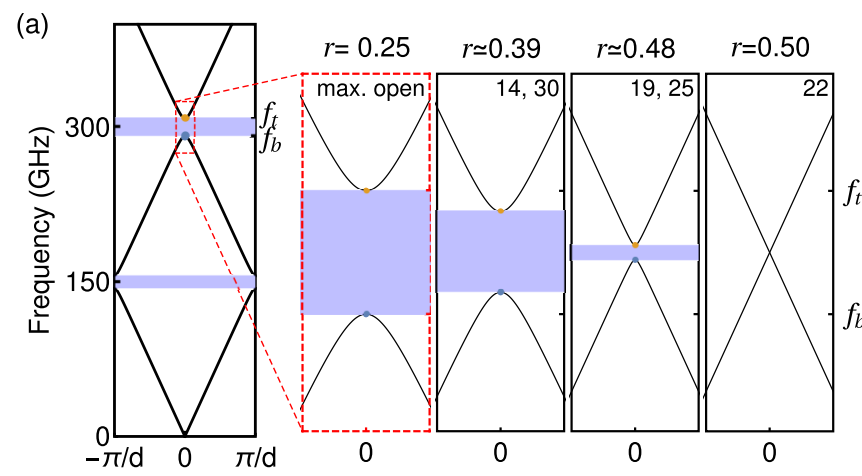

Wavevector q

(b)
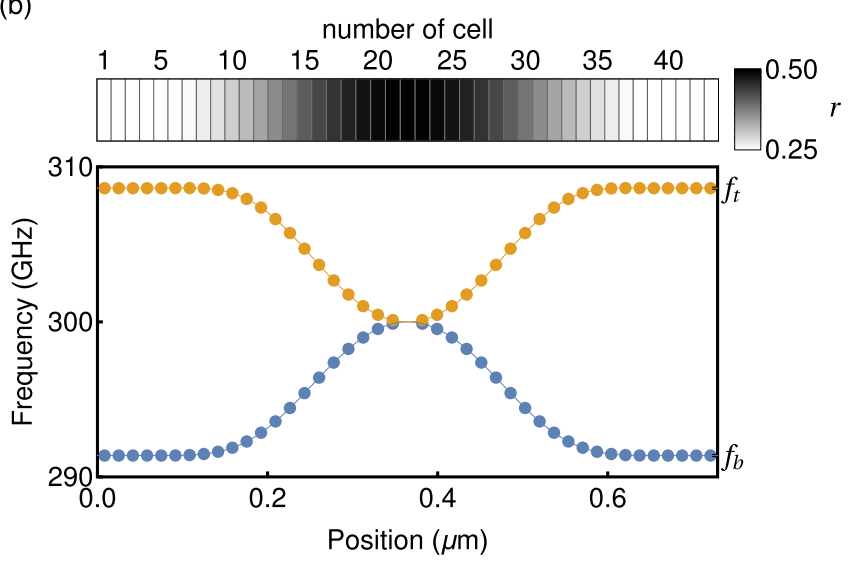

FIG. 1. (a) Left: Acoustic band diagram of a superlattice with its second minigap maximally opened. The first two acoustic minigaps at 150 and $300 \mathrm{GHz}$ are highlighted in light blue. They correspond to the first minigaps at the Brillouin zone edge and center, respectively. $f_{b}$ and $f_{t}$ mark the bottom and top edge of the second minigap. Right: Zoom-in of the acoustic band structure around the second acoustic minigap (dashed red rectangle) associated with the unperturbed cells, cells 14 and 30, 19 and 25, and 22, respectively. $r$, the relative acoustic path length of AlAs in the unit cell, is depicted on top of each panel. (b) Top: Schematic of an adiabatic perturbation induced in a periodic structure of 43 unit cells. The grayscale indicates the value of $r$ for each cell. Its modulation follows a $\cos ^{2}$ shape between cells 6 and 38 with an amplitude of 0.25 while remaining constant at 0.25 in the rest of the structure. Layer thicknesses for cells with $r=0.25$ are $4.72 \mathrm{~nm}$ and $11.95 \mathrm{~nm}$ for AlAs and GaAs layers, respectively. Along the modulated region the thicknesses remain close to those values. Bottom: Local acoustic band structure representing the minigap edges for each cell as a function of position. Each vertically aligned pair of dots corresponds to the minigap edges associated with each cell and plotted at each cell center. The curves mark the continuous trajectory targeted for each edge.

a maximally opened minigap at $r=0.25$ (first panel), the gap completely vanishes for a value of $r=0.5$, i.e., when the two material layers have equal acoustic thickness. This holds for the second minigap. In general, for the $n$th gap, the gap is closed for $n+1$ values of $r$. We use this dependence to construct a first example of an effective phonon potential by adiabatically varying the parameter $r$ in a multilayer structure. Starting from a structure composed of 43 unit cells with $f_{d}=300 \mathrm{GHz}, D=1$, and $r=0.25$, the potential is 
(a)

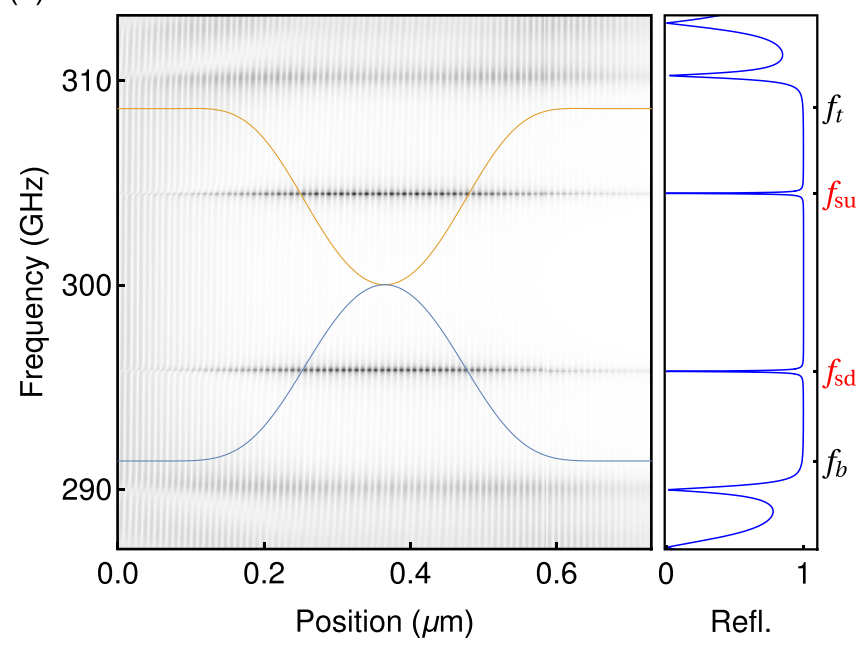

(b)
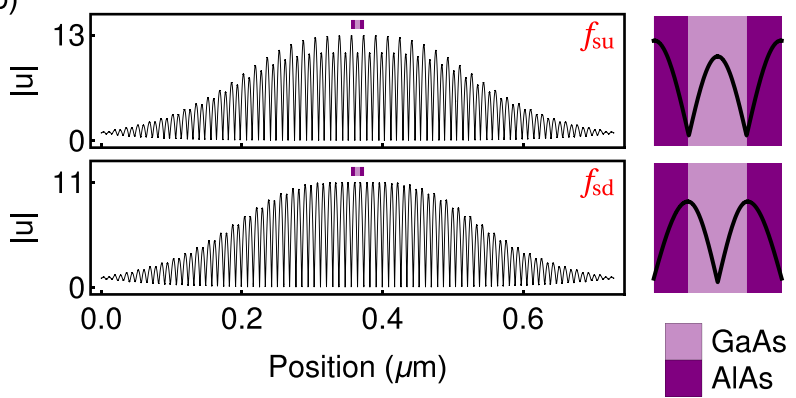

FIG. 2. (a) Right: Acoustic reflectivity of an adiabatic resonator created by introducing a modulation in the ratio of AlAs per unit cell in a periodic structure. $f_{b}$ and $f_{t}$ mark the maximally opened stopband limits while $f_{s d}$ and $f_{s u}$ are the frequencies of the confined modes. Left: Displacement distribution as a function of position and frequency for phonons propagating from left to right. (b) Left: Normalized displacement profile of the confined modes. Right: For each mode, a zoom-in of the profile at the central cell is shown.

designed by modulating $r$ following a $\cos ^{2}$ pattern [5] along the 33 central unit cells [see top part of Fig. 1(b)]. There, $r$ first increases up to $r=0.5$ at the central cell and then changes back to the initial value of $r=0.25$. By doing so, the width of the second minigap undergoes a closing and reopening along the structure. The term adiabatic refers to the condition that the considered minigap shows a large overlap between consecutive unit cells. In this work, our structures have typical overlaps beyond $93 \%$. The corresponding evolution of the upper and lower band edges is shown in the bottom of Fig. 1(b). Here we have adopted the notion of a "local band structure" [5,22,31-33], i.e., each unit cell of the structure is assigned the bands of an infinite superlattice having the same structural parameters. The concept of a local band structure for acoustic phonons in superlattices is similar to the emergence of conduction minibands for electrons in short period superlattices [34]. By engineering the local band structure we control the group velocity of the acoustic phonons.

To illustrate how a multilayered structure acts as an effective phononic potential, we embed it in a GaAs continuum and calculate its acoustic reflectivity spectrum shown in the right panel of Fig. 2(a) using a transfer matrix formalism. The displayed spectrum presents a central high reflectivity band containing two sharp reflectivity dips marked $f_{s d}$ and $f_{s u}$. Outside this region the reflectivity shows Bragg oscillations, a common feature in superlattices $[6,35]$. The high reflectivity region corresponds to the frequency range of the maximally open minigap $f_{b}<f<f_{t}$, where the structure acts as a distributed Bragg reflector (DBR). The two dips correspond to two resonances of the structure. Their mechanical displacement profiles $u(z)$ along the superlattice are plotted in Fig. 2(b). Both states are confined to the modulated minigap region with exponentially decaying tails inside the unmodulated outer parts of the structure. In an earlier report, an adiabatic nanoacoustic cavity was presented [5] in which the width of a band gap was kept constant and an effective potential well was obtained by progressively shifting the gap towards lower frequencies along the structure. In contrast to this case, we here kept the central frequency of the gap constant and changed its width along the structure. We furthermore compute the mechanical displacement within the structure upon an incident plane wave of unit amplitude as a function of frequency and position. The plot is superimposed with the $\cos ^{2}$ evolution of the local band edges (blue and orange lines) in Fig. 2(a) (left panel). The modulation of $r_{\mathrm{AlAs}}$ in the central region closes and reopens the local minigap. This produces a wasp-waist shaped local band structure. The two confined modes at $f_{s d}$ and $f_{s u}$ are evidenced by a strongly enhanced mechanical response in the modulated central part of the superlattice. The superlattice resembles the behavior of a quantum-mechanical potential well with bound, localized solutions at energies below the band edge and a continuum of propagating solutions for energies outside the maximally open band gap. In analogy to a quantum mechanical potential well, the number of bound states increases if the well is widened or deepened. Interestingly, the potential well studied here supports two confined states, one in its upper convex dip at frequency $f_{s u}$, the other in the lower concave part at a frequency $f_{\text {sd }}$. While both states present similar envelopes, their overall spatial symmetry of the carrier is opposite [see the zoom-in of $|u|$ along the central cell at the right of each panel in Fig. 2(b)]. This is a direct consequence of the different spatial symmetries of the Bloch modes at the upper and lower band edge [27,36,37] [marked yellow and blue in Fig. 2(a), respectively]. These states are the equivalent of a bound electronic state and a bound hole. This observation represents a new aspect of adiabatic cavities compared to earlier works where only single-sided potential wells were considered [5,31]. In the rest of the paper we will extend the concept of phononic band engineering to generalized acoustic potentials.

\section{EFFECTIVE POTENTIALS}

We have shown how to achieve a double-sided acoustic potential well by closing and reopening a minigap. Extending this approach for general potentials requires us to modulate both the spectral width of a phononic minigap and its central frequency. The latter is achieved by scaling the overall thickness of the unit cell. To this end, we rewrite the dispersion 
relation of a bilayer superlattice as

$$
\begin{aligned}
1= & \cos \left(\frac{2 \pi f_{b}}{f_{d}}\right) \\
& -\frac{\epsilon^{2}}{2} \sin \left(\frac{2 \pi f_{b} r}{f_{d}}\right) \sin \left(\frac{2 \pi f_{b}(1-r)}{f_{d}}\right) .
\end{aligned}
$$

Here we have set $q=0$ since we target the second minigap at the center of the Brillouin zone. We have furthermore set $f=$ $f_{b}$ and $D=1$. This allows us to shape the local band structure by placing its bottom edge at a fixed value $f_{b}$ while controlling the top edge at will through varying $f_{d}$ and solving Eq. (4) for $r$. Next, we will illustrate this approach by designing three structures mimicking a parabolic, a Morse, and a double-well potential. These potentials constitute three textbook examples with well known solutions which allow us to make a detailed comparison between the electronic and phononic behaviors.

\section{A. Parabolic potential}

As a first case we study a parabolic potential. This potential is usually associated with a harmonic oscillator with equidistant energy levels. While the quadratic dispersion relation of electrons indeed results in eigenvalues following a linear dependence with respect to the mode number $n$, the linear dispersion relation of phonons in contrast results in a dependence proportional to $n^{2 / 3}$ [22] mimicking the case of relativistic electrons or light. In the optical domain, similar effective potentials have been demonstrated using two-dimensional photonic crystal structures [38,39]. In nanoacoustics a similar potential has been proposed using coupled acoustic nanocavities $[21,22]$.

Using Eq. (4), we design an acoustic structure mimicking a parabolic potential of the form

$$
V(x)=V_{0} x^{2} \text {. }
$$

The variable $x$ is a unitless, normalized position varying from -1 to 1 from the leftmost to the rightmost unit cell of the structure. The amplitude of the potential is chosen as $V_{0}=$ $f_{t}-f_{b}$ such that its value ranges from a maximally opened minigap at the first and last unit cell to a closed gap at the center. Note that both $V(x)$ and $x$ vary in discrete steps from cell to cell along the structure. To obtain an effectively smooth potential, we choose a structure composed of $N=101$ cells and numerically solve the $N$ equations to obtain the parameter $r$ defining the local band structure for each cell.

Figure 3(a) (right panel) presents the acoustic reflectivity for this structure, where we observe a series of dips associated with the confined modes in the acoustic potential. The modes labeled $f_{b}$ and $f_{t}$ indicate the superlattice stopband edges for the maximally opened minigap. The modes labeled $f_{1}$ to $f_{4}$ correspond to the first four bound states. The left panel in Fig. 3(a) shows the local band structure on top of the displacement distribution color map calculated in the same manner as in Fig. 2(a). Modes $f_{1}$ to $f_{4}$ are localized inside the parabola similarly to what was observed in the previously discussed potential well. The observed unequal spacing between modes follows the expected $n^{2 / 3}$ dependence. Figure 3(b) shows the displacement profile corresponding to an incident plane wave of unit strength for each of the first four modes.

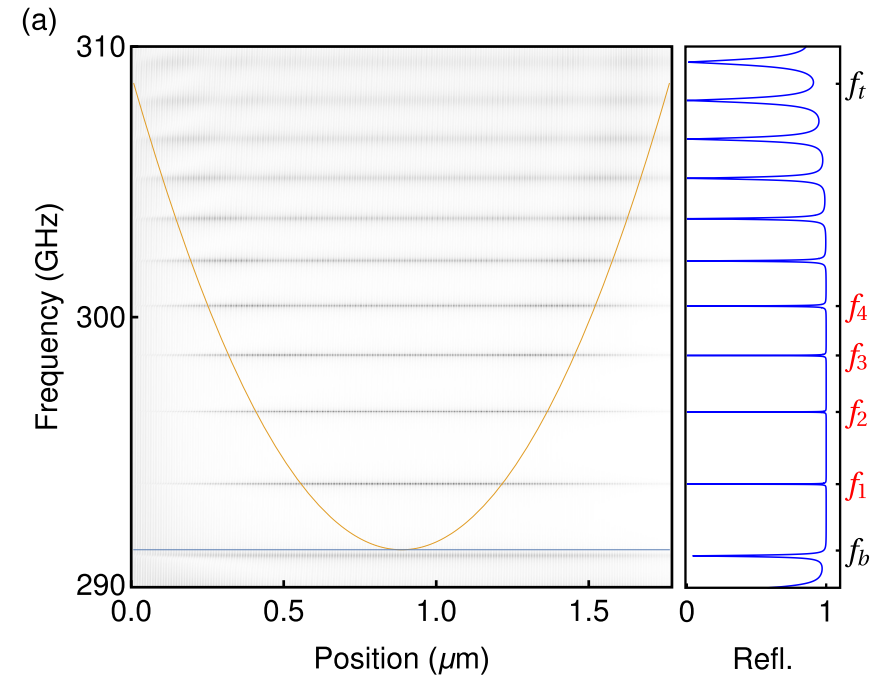

(b)

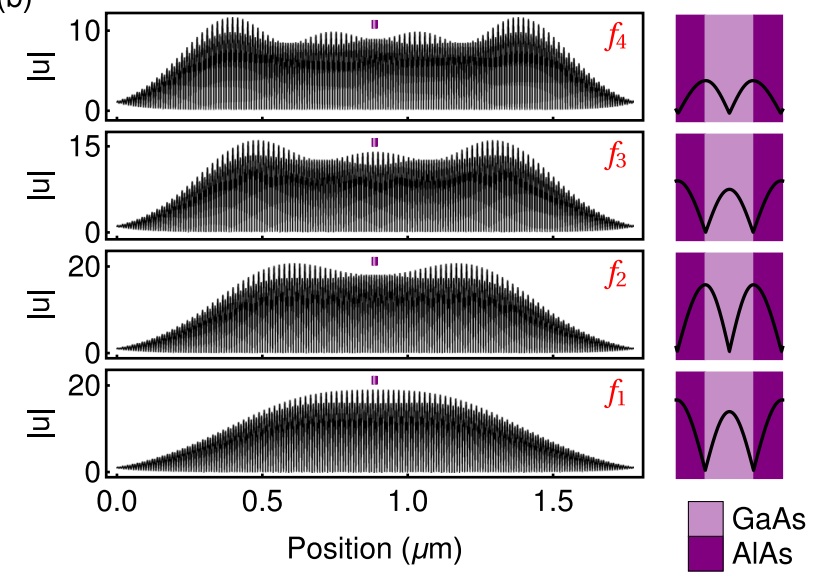

FIG. 3. (a) Right: Acoustic reflectivity for the parabolic potential structure. $f_{b}$ and $f_{t}$ mark the DBR stopband limits while $f_{1}$ to $f_{4}$ mark the first four confined modes. Left: Displacement distribution as a function of position and frequency for phonons propagating from left to right. On top the local band structure is plotted. (b) Left: Normalized displacement profile of the first four confined modes. Right: For each mode, a zoom-in of the profile at the central cell is shown.

They are mainly localized in the center of the structure with two evanescently decaying tails on the sides. Their envelopes exhibit $n$ maxima for the $n$th confined mode. For the first four modes, notice that the length of the evanescent tails decreases with increasing order. This can be explained by considering that the penetration depth for phonons at frequencies within a DBR stopband not only depends on the minigap bandwidth but also on the spectral position within the gap. For a given width of the gap, the evanescent decay length of a mode is the shortest if it lies at the band gap center and diverges when approaching the gap edges. Therefore, the decay length of the modes in the parabolic potential decreases with ascending order since the modes appear closer to the band gap center. For further analysis, each confined mode profile in Fig. 3(b) includes a zoom-in of its central cell depicted at the right of each panel. The central cell position and size are also represented as insets. As for the acoustic potential 
well presented in the previous section, the fundamental mode confined at $f_{1}$ exhibits the same overall spatial symmetry as the Bloch mode at the upper band edge. For the higher order modes we, however, observe alternating symmetry properties in agreement with the well-known eigenstates of the quantummechanical harmonic oscillator [34].

\section{B. Morse potential}

The proposed method enables us to implement potentials that are asymmetric in space such as the Morse potential. This potential is used to model interatomic interactions in diatomic molecules. It presents a well shape but it distinguishes itself from the quantum harmonic oscillator by an asymptotic limit in the potential energy on one side. By doing so, it includes the effect of chemical bond breaking that the quantum harmonic oscillator does not consider. The resulting modes of such a potential are either bound below the asymptotic energy limit or unbound above it.

The Morse potential as a function of a radial coordinate $r$ can be expressed as

$$
V(r)=V_{0}\left\{\frac{1-\exp \left[a\left(r_{e}-r\right)\right]}{1-\exp \left[a r_{e}\right]}\right\}^{2},
$$

with the parameters $V_{0}, a$, and $r_{e}$ determining the depth, width, and equilibrium position of the potential, respectively. The variable $r$ is a unitless, normalized position along the structure varying from 0 to 1 from the leftmost to the rightmost unit cell. We set $a \approx 3.95$ and $r_{e} \approx 0.26$ while choosing $V_{0}=f_{t}-f_{b}$, i.e., the potential spans the full range from a completely closed to a fully opened acoustic minigap.

Figure 4(a) (right panel) presents the acoustic reflectivity of such a structure composed of 201 unit cells. As before, $f_{b}$ and $f_{t}$ correspond to the edges of the maximally opened minigap. Two main bands can be distinguished; a high reflectivity band where three modes appear (labeled $f_{m 1}, f_{m 2}$, and $f_{m 3}$ ) and a higher energy band with reflectivity oscillations. In contrast to the parabolic potential, which showed marked reflectivity dips, the modes of the Morse potential are associated with shallow reflectivity dips. This can be explained by considering that the structure is asymmetric, similar to an unbalanced Fabry-Perot resonator [40], i.e., a structure effectively composed of two mirrors with unequal reflectivity. The imbalance causes predominant decay of the confined modes to one side and hence limits the achievable minimum reflectivity upon interference of multiple internal reflections. The left panel in Fig. 4(a) shows the local band structure on top of the displacement color map in response to a plane wave of unit strength incident from the left. We see the confined modes corresponding to the bound states within the dip of the Morse potential for frequencies below $f_{\text {esc }}$. Above this frequency, a series of unbound modes appears corresponding to the oscillations in the reflectivity plotted in Fig. 4(a) (right panel). Finally, Fig. 4(b) shows the displacement profile of the first three confined modes. Comparing these with those of Fig. 3(b), we see that the number of maxima in the envelope corresponds to the order of the mode. In the case shown here, the left and right evanescent tails are different. Once again, we show the symmetric/antisymmetric alternation between consecutive confined modes as depicted in the central cells at
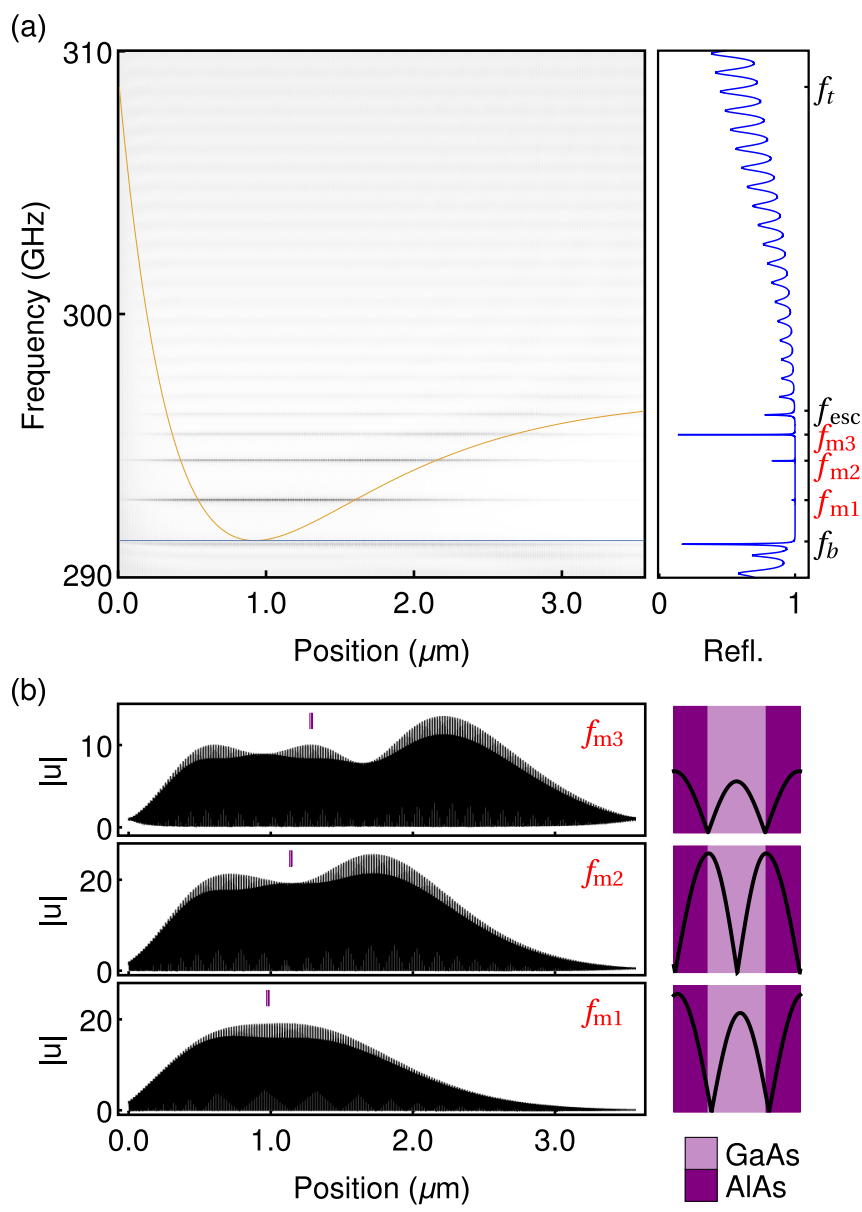

FIG. 4. (a) Right: Acoustic reflectivity for the Morse potential structure. $f_{b}$ and $f_{t}$ mark the DBR stopband limits while $f_{m 1}, f_{m 2}$, and $f_{m 3}$ the first three confined modes. Left: Displacement distribution as a function of position and frequency for phonons propagating from left to right. Local band structure plotted on top. (b) Left: Normalized displacement profile of the first three confined modes. Right: For each mode, a zoom-in the profile at the central cell of the mode is shown.

the right of each panel in Fig. 4(b). Since the structure is not symmetric, we define the central cell for each confined mode as the cell with the central maximum or minimum of the mode envelope.

\section{Double-well potential}

Based on the same design principles, we engineer the phononic equivalent of a hydrogen molecule. In the context of quantum mechanics, it is the result of the hybridization of degenerate eigenstates of two individual quantum wells, similar to the one presented in Sec. III A. This double-well potential thus has eigenstates that extend over two well-defined regions of space separated by a tunneling barrier. By modifying the barrier, it is possible to control the coupling strength and hence the energy splitting of the hybridized modes. Note that this type of potential can also be reasoned with a tight-binding approach, i.e., each of the two atoms is represented by an individual parabolic potential, and the hopping term by an effective transmission through the barrier [22]. 
Previous realizations of acoustic molecules have been based on coupling two cavities $[21,22]$. By customizing the shape of the double well in the way presented here, we gained additional control on the envelope of the confined modes. Furthermore, each isolated phononic atom supports multiple modes besides the fundamental one. In this way, the energy and the splitting of the hybridized modes can be engineered. The optical counterpart of this systems, known as photonic molecules [41], appears as a promising platform for the development of quantum technologies and could inspire future phononic technologies $[42,43]$.

The potential energy as a function of the parameter $z$ for a double-well potential can be expressed as

$$
V(z)=V_{0}\left[\left(\lambda z^{4}-k z^{2}\right)+k^{2} / 4 \lambda\right],
$$

where $\lambda$ and $k$ are both positive parameters determining the position and depth of both wells. The variable $z$ is the unitless, normalized position along the structure varying from -1 to 1 from the leftmost to the rightmost unit cell. We choose $\lambda \approx$ 2.39 and $k \approx 1.69$ such that it spans values from 0 to $V_{0}=$ $f_{t}-f_{b}$.

Figure 5(a) (right panel) shows the acoustic reflectivity of a double-well structure built of 201 unit cells. The top edge frequency at the central hump is labeled $f_{\text {bar }}$, while $f_{b}$ and $f_{t}$ correspond to the edges of the maximally opened minigap. The oscillations above $f_{\text {bar }}$ correspond to modes localized in the full structure. Below $f_{\text {bar }}$ a high reflectivity region with four dips appears. These are grouped in pairs and labeled $f_{1 s}$, $f_{1 a}, f_{2 s}$, and $f_{2 a}$. Figure 5(a) (left panel) shows the local band structure on top of the displacement color map. We can see how bound modes below $f_{\text {bar }}$ are simultaneously localized in both potential wells. The central hump separating these two wells acts as a potential barrier between them. Tunneling through it, acoustic phonons propagate from one well to the other. Consequently, increasing or reducing the barrier will increase or reduce the coupling between the wells. Figure 5(b) shows the displacement profiles of the first four resonant modes. We can see how the confined modes span along all the structure with a small amplitude in the central hump, in particular, for the first pair resonances at $f_{1 s}$ and $f_{1 a}$. For resonances at $f_{2 s}$ and $f_{2 a}$ the closeness to the top of the barrier raises the amplitude in the center as confinement transits from localization in the two wells towards localization in all the structure. Each pair shares a similar shape of the envelope. A more detailed analysis however evidences the hybridization into symmetric and antisymmetric solutions. At the right side of each panel a zoom-in of the displacement profile at the central cell is presented. As before, we can see how the first mode shares the symmetry of its enclosing band edges, while the second mode has the opposite total symmetry. As in the other potentials, the number of maxima in the mode envelopes scales with the order of the mode pair. In resemblance with the Morse case, we can see the envelope not being symmetric with respect to each well but increasing towards the center of the structure. This results from each confinement region (well) being surrounded by unbalanced reflective regions as in the Morse potential case.

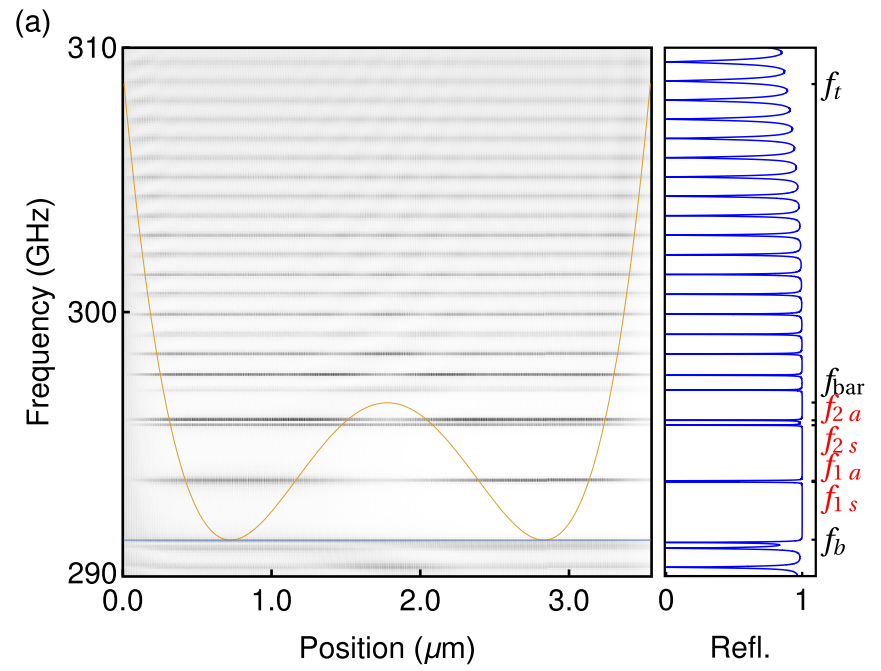

(b)

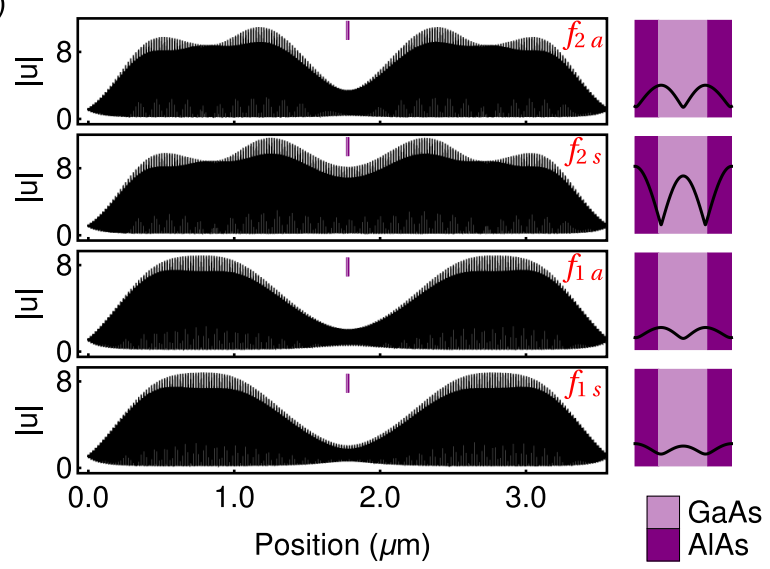

FIG. 5. (a) Right: Acoustic reflectivity for the double-well potential structure. $f_{b}$ and $f_{t}$ mark the DBR stopband limits while $f_{1 s}, f_{1 a}$, $f_{2 s}$, and $f_{2 a}$ mark the first four confined modes. Left: Displacement distribution as a function of position and frequency for phonons propagating from left to right. Local band structure plotted on top. (b) Left: Normalized displacement profile of the first four confined modes. Right: For each mode, a zoom-in of the displacement profile at the central cell is shown.

\section{PHONON POTENTIALS INCLUDING LOSS}

In the frequency range of hundreds of $\mathrm{GHz}$, acoustic phonons are subject to loss mechanisms [20,44-46] that might influence the performance and experimental feasibility of the phonon engineering concepts outlined above. Here we extend our analysis by including the effect of phonon attenuation in our transfer matrix simulations and study the ensuing impact on spatial confinement, quality factors, and reflectivity spectra for the studied nanomechanical structures. For realistic losses we find that the effective picture of phonon potentials presented in our paper holds.

In order to preserve the sharp acoustic resonances found in our simulations, e.g., for the harmonic oscillator potential (cf. Fig. 3), phonons need to propagate back and forth along the structure hundreds of times. That this condition is indeed satisfied under realistic conditions is substantiated by propagation lengths for longitudinal acoustic phonons 
in GaAs reported in previous works [20,47]. Propagation lengths up to millimeters were found at low temperatures for longitudinal acoustic phonons in the hundreds of $\mathrm{GHz}$ range. The structures considered here are around two orders of magnitude smaller (biggest structure is $\sim 3.5 \mu \mathrm{m}$ thick), hence allowing confined modes to build up under realistic losses. To substantiate this claim, we have extended our transfer matrix simulations and carefully revisit in particular the cases of the harmonic oscillator and the double-well potential since they present high quality factors and small mode spacing, respectively. These structures are hence expected to be most susceptible to the consequences of even small amounts of phonon losses.

Acoustic phonon losses can be introduced as an acoustic extinction coefficient $\kappa_{\mathrm{ac}}$ in an analogous way to the optical extinction coefficient $\kappa$ (i.e., the imaginary part of the index of refraction) appearing in the optical wave vector

$$
k_{\mathrm{op}}=2 \pi \frac{n+i \kappa}{\lambda_{\mathrm{op}}} .
$$

Here $\underline{n}=n+i \kappa$ is the complex index of refraction and $\lambda_{\text {op }}$ is the optical wavelength in vacuum. The acoustic wave vector is defined as

$$
k_{\mathrm{ac}}=2 \pi \frac{1+i \kappa_{\mathrm{ac}}}{\lambda_{\mathrm{ac}}},
$$

where $\lambda_{\mathrm{ac}}$ is the acoustic wavelength in the medium. This approach has been used, e.g., in $[46,48,49]$ and it has been shown that phonon extinction coefficients can be derived from the corresponding phonon viscosity tensor [50]. While $\kappa_{\mathrm{ac}}$ by itself is sufficiently general to quantify the losses experienced by phonons propagating in a medium, it is useful to establish the relationship between the acoustic extinction coefficient and more comprehensive quantities such as the acoustic penetration depth $\delta_{\mathrm{ac}}$. For phonons in a given material at a frequency $f$ it is given by

$$
\delta_{\mathrm{ac}}=\frac{\lambda_{\mathrm{ac}}}{4 \pi \kappa_{\mathrm{ac}}} .
$$

This definition implies that for a penetration depth of $\delta_{\mathrm{ac}}$, the intensity of a propagating phonon (i.e., square of the displacement amplitude $|u|^{2}$ ) is reduced by a factor of $1 / e$ after traveling a distance $\delta_{\mathrm{ac}}$. For GaAs it has been found that longitudinal acoustic phonons in the frequency range of $300 \mathrm{GHz}$ (as considered in our simulations) propagate over distances as large as $\delta_{\mathrm{ac}} \sim 1 \mathrm{~mm}$ at cryogenic temperatures $[20,47]$.

With the implementation of losses in our transfer matrix formalism at hand, we revisit the parabolic potential resonator. This symmetric structure has 201 unit cells and presents particularly high quality factors (max. $Q \sim 30000$ in the lossless case). Therefore, the effect of losses should be most noticeable for this potential. We proceed to analyze the effect of losses by computing quality factors as a function of the acoustic penetration depth $\delta_{\mathrm{ac}}$ for the first four bound acoustic modes at frequencies $f_{1}, f_{2}, f_{3}$, and $f_{4}$. The results are shown in Fig. 6(a). In the underlying calculation we have assumed equal acoustic extinction coefficients for all layers of the structure. We find that for penetration depths below $200 \mu \mathrm{m}$ the $Q$ factors rapidly decrease. Nevertheless, even for the sharpest (a)

(b)
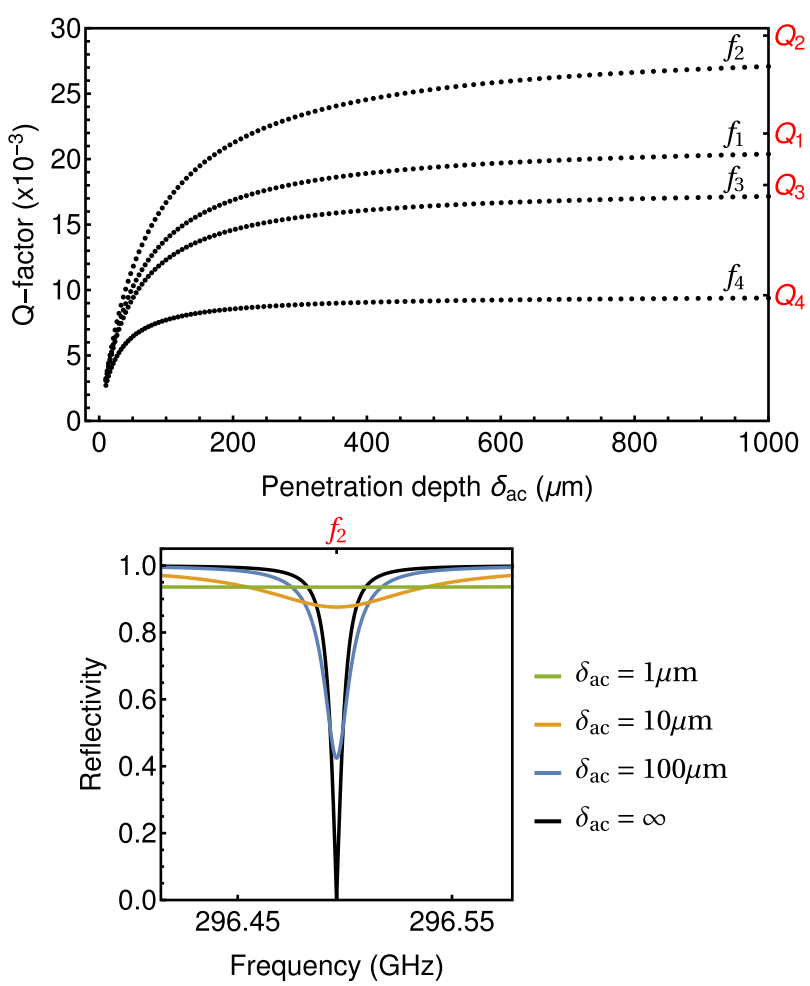

FIG. 6. (a) $Q$ factors of the four lowest confined modes of the parabolic nanophononic potential (see Fig. 3) if losses are included via a finite phonon penetration depth $\delta_{\mathrm{ac}}$. For experimentally realistic penetration depths $\delta_{\mathrm{ac}} \approx 300 \mu \mathrm{m}$ the $Q$ factors change by less than $8 \%$. The quality factors in the lossless case are indicated with labels $Q_{1-4}$ on the right axis. (b) Acoustic amplitude reflectivity spectra in the close vicinity of the confined mode with frequency $f_{1}$. With loss increasing from zero $\left(\delta_{\mathrm{ac}}=\infty\right)$, the contrast of the reflectivity dip diminishes. However, only for extremely high losses around $\delta_{\mathrm{ac}}=$ $1 \mu \mathrm{m}$ the resonance is completely washed out.

resonance at $f_{2}$, the $Q$ factor still remains beyond 20000 for a penetration depth of $200 \mu \mathrm{m}$.

We have also calculated corresponding reflectivity spectra for the resonance at $f_{2}$ when varying the acoustic penetration depth over several orders of magnitude from $1 \mu \mathrm{m}$ to $100 \mu \mathrm{m}$. The results are shown in Fig. 6(b) together with the lossless case for reference $\left(\delta_{\mathrm{ac}}=\infty\right)$. We find that due to the losses the sharp reflectivity dips get wider and no longer drop to zero but a finite reflectivity persists. For experimentally realistic losses in the range of $\delta_{\mathrm{ac}}=300 \mu \mathrm{m}$ in GaAlAs for phonons at frequencies around $300 \mathrm{GHz}$ even the sharpest mode at $f_{2}$ thus stays clearly visible. Only for extremely strong losses in the the range of $\delta_{\mathrm{ac}}=1 \mu \mathrm{m}$ the resonance gets completely washed out. This persistence of the modes is a direct consequence of the small structure thickness achieved by phonon engineering with individual superlattices. Alternative implementations of these kinds of potentials, e.g., with coupled acoustic resonators [25], would be much more susceptible to losses since the required structure thicknesses are orders of magnitude larger.

For comparison, we also study the effects of losses in the double-well potential resonator. Because this resonator 
(a)

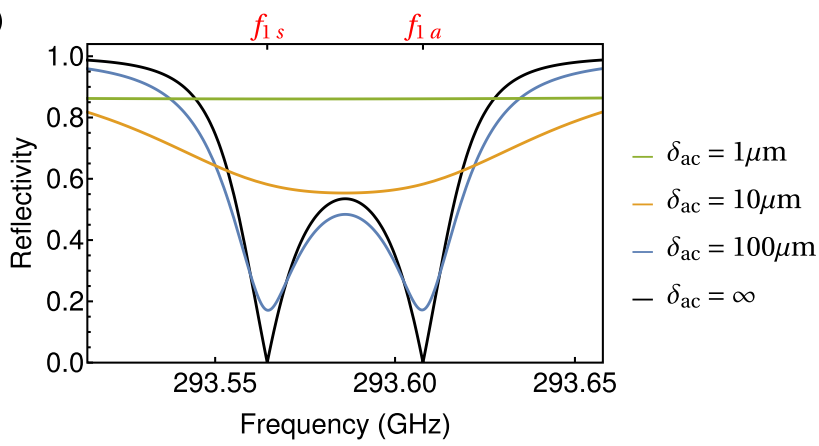

(b)

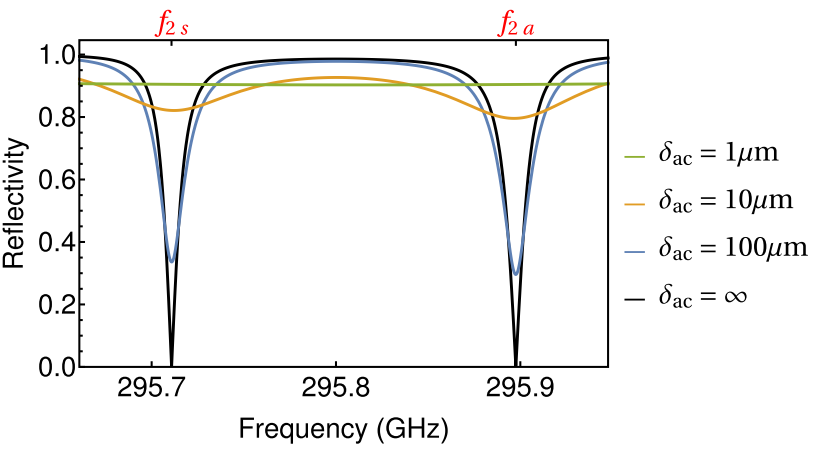

FIG. 7. (a) Reflectivity dips associated with resonances of the phononic double-well potential at $f_{1 s}$ and $f_{1 a}$ for different penetration depths. (b) Same as in (a) for resonances at $f_{2 s}$ and $f_{2 a}$.

presents hybridized confined modes, resonant frequencies localized in the two wells (i.e., below $f_{\text {bar }}$ ) appear in pairs with small frequency spacing (e.g., $f_{1 a}-f_{1 s} \approx 43 \mathrm{MHz}$ ). While for the parabolic potential resonator modes remain discernible for penetration depths down to $\delta_{\mathrm{ac}}=10 \mu \mathrm{m}$, the small frequency separation in the double-well case affects the possibility to distinguish between nearby modes. Zoom-ins to the reflectivity spectra for various penetration depths are displayed in Fig. 7. Displayed are the frequency intervals around the resonances at frequencies $f_{1 s}$ and $f_{1 a}$ [Fig. 7(a)] and at frequencies $f_{2 s}$ and $f_{2 a}$ [Fig. 7(b)]. For the second pair of hybridized modes each resonance remains clearly distinguishable even for short decay lengths around $\delta_{\mathrm{ac}}=$ $10 \mu \mathrm{m}$. Only for extremely large phonon losses corresponding to $\delta_{\mathrm{ac}}=1 \mu \mathrm{m}$, the resonances are completely washed out. For the first pair of hybridized modes, the initial mode splitting is smaller leading to a partial overlap between the reflectivity dips even in the lossless case. Nevertheless, the two separate reflectivity minima remain discernible down to penetration depths of $100 \mu \mathrm{m}$, that is, well below the experimentally realistic case. It is worth noting that in all the cases considered in this discussion we neglected losses due to the roughness at the interfaces between layers of different materials. These additional losses scale with the number of layers [17] in contrast to propagation losses, which are governed by the total thickness of the structure under consideration.

\section{DISCUSSION AND CONCLUSIONS}

We have demonstrated how a local engineering of the group velocity in nanoacoustic superlattices can be used for the implementation of arbitrary effective potential landscapes for longitudinal acoustic phonons. First, by introducing the concept of a local band structure, we have designed an adiabatic potential well resonator, and shown how it confines acoustic modes mimicking electron and hole states. Second, we have parametrized the dispersion relation of bilayer superlattices isolating parameters determining the minigap bandwidths and positions. Using this parametrization, three potentials have been studied: a parabolic, a Morse, and a double-well potential. While the main features of the electronic counterparts are reproduced, effective phonon potentials present significant novel aspects to remark. The spatial profile of the confined modes (in particular its decay length towards the exterior) depends on the position and frequency dependent penetration depth as well as the boundary conditions. For the Morse potential the dips in the reflectivity curve corresponding to the confined modes are shallow as a consequence of confinement between unbalanced effective phonon mirrors. Finally, the double-well potential highlights the role of the symmetries of the spatial mode profiles in hybridized mode solutions. In all three cases, the spectrum of confined modes is fundamentally different from the standard electronic counterpart, since the phonon dispersion relation in a bulk material is linear rather than parabolic. The systems described here correspond to the dynamics of quasirelativistic particles in potential wells which have a linear dispersion [22,51].

The potentials are implemented by smoothly changing the thicknesses of the layers forming each unit cell in a superlattice. The full structure is typically formed by approximately 200 unit cells. Layer thicknesses vary between 4.72 and $11.95 \mathrm{~nm}$ for all the presented structures, corresponding to resonance frequencies around $300 \mathrm{GHz}$. From a practical point of view, such lattices can be experimentally realized in molecular beam epitaxy (MBE) growth [52]. In comparison, relying on coupled cavities to study phonon dynamics rather than single superlattices, an equivalent implementation of the potentials presented in this work would require materials with higher impedance contrast or very thick samples, which are much more demanding to grow [21]. A detailed analysis of the effect of losses in the structures has shown that under realistic conditions the proposed potential resonators would still exhibit the same main features presented for the lossless case. This ability to preserve rich phonon dynamics in intricate potential landscapes even under the influence of losses is in particular due to the small overall thickness of the structures compared to implementations of phonon potentials with coupled acoustic cavities. However, the role of roughness in the interfaces might play a role in the experimental feasibility of these structures.

The selection of the first zone-center minigap is essential for our implementation of effective phononic potentials, since it can be completely closed in a nontrivial way by changing the internal unit cell structure [27]. Compared to higher order minigaps, which also allow a complete nontrivial closure, the second minigap width is least susceptible to fabrication errors since its variation with the ratio of relative acoustic path length in the lattice unit cell $r$ is slower. This makes the implementation of the proposed structures more realistic from an experimental point of view. Both zone center and zone 
edge modes are in principle accessible in coherent phonon generation and detection experiments or Brillouin scattering $[27,29,53]$.

A necessary condition in both approaches is that the system allows a description in terms of local band structures. Here we ensured this by keeping the overlap between the second minigap of consecutive unit cells sufficiently large. As a consequence, the shape of the potential landscape to be mimicked will impose a minimum number of layers needed depending on its maximum gradient. By implementing potentials with a single superlattice it is thus possible to experimentally realize potentials that are unrealistic with coupled nanocavities. Moreover, key results that were already achieved based on the tight-binding approach with coupled cavities [22], such as phonon molecules and Bloch oscillations in Wannier-Stark ladders, could be also implemented through the nearly free electron approach employed here [21]. Similarly, the material composition [28] of each layer or alternating crystal phases [54] could be used as a parameter to engineer the local phonon group velocity.

The materials GaAs and AlAs used in our simulations present the unique feature that their acoustic impedance contrast and index of refraction contrast are nearly the same. As a result, cavities simultaneously confining both sound and light have been achieved [53,55-58]. Exploiting this feature, the design procedure for general potential landscapes introduced here could be extended to control simultaneously the propagation of phonons and photons in optomechanical systems.

Another fundamental property of acoustic superlattices that has not been exploited here are the Bloch mode symmetries at the minigap edges. It has been shown [59] that an inversion of these symmetries can be achieved by closing and reopening a minigap, inducing a topological phase transition and hence allowing the construction of topological interface states [26-29]. This approach could for example be combined with the adiabatic resonator presented in Fig. 2, resulting in an additional confined state between the two modes of the well, i.e., mimicking a zero-energy state between electron and hole. Overall, we presented a series of acoustic devices allowing the control of the propagation of longitudinal acoustic phonons that are achievable through standard MBE growth and can be tested using optical pump-probe and Brillouin scattering schemes $[18,60]$.

\section{ACKNOWLEDGMENTS}

The authors thank F. R. Lamberti, P. Senellart, and L. Lanco for fruitful discussions. The authors acknowledge funding by the European Research Council Starting Grant No. 715939, Nanophennec. M.E. acknowledges funding by the Deutsche Forschungsgemeinschaft (DFG, German Research Foundation) Project 401390650.
[1] S. Volz, J. Ordonez-Miranda, A. Shchepetov, M. Prunnila, J. Ahopelto, T. Pezeril, G. Vaudel, V. Gusev, P. Ruello, E. M. Weig, M. Schubert, M. Hettich, M. Grossman, T. Dekorsy, F. Alzina, B. Graczykowski, E. Chavez-Angel, J. Sebastian Reparaz, M. R. Wagner, C. M. Sotomayor-Torres, S. Xiong, S. Neogi, and D. Donadio, Nanophononics: State of the art and perspectives, Eur. Phys. J. B 89, 15 (2016).

[2] A. A. Balandin, Nanophononics: Phonon engineering in nanostructures and nanodevices, J. Nanosci. Nanotechnol. 5, 1015 (2005).

[3] A. A. Balandin, E. P. Pokatilov, and D. L. Nika, Phonon engineering in hetero- and nanostructures, J. Nanoelectron. Optoelectron. 2, 140 (2007).

[4] N. D. Lanzillotti-Kimura, B. Perrin, A. Fainstein, B. Jusserand, and A. Lemaître, Nanophononic thin-film filters and mirrors studied by picosecond ultrasonics, Appl. Phys. Lett. 96, 053101 (2010).

[5] F. R. Lamberti, M. Esmann, A. Lemaître, C. Gomez Carbonell, O. Krebs, I. Favero, B. Jusserand, P. Senellart, L. Lanco, and N. D. Lanzillotti-Kimura, Nanomechanical resonators based on adiabatic periodicity-breaking in a superlattice, Appl. Phys. Lett. 111, 173107 (2017).

[6] M. Trigo, A. Bruchhausen, A. Fainstein, B. Jusserand, and V. Thierry-Mieg, Confinement of Acoustical Vibrations in a Semiconductor Planar Phonon Cavity, Phys. Rev. Lett. 89, 227402 (2002).

[7] N. Lanzillotti-Kimura, A. Fainstein, and B. Jusserand, Towards $\mathrm{GHz}-\mathrm{THz}$ cavity optomechanics in DBR-based semiconductor resonators, Ultrasonics 56, 80 (2015).
[8] C. Brüggemann, A. V. Akimov, A. V. Scherbakov, M. Bombeck, C. Schneider, S. Höfling, A. Forchel, D. R. Yakovlev, and M. Bayer, Laser mode feeding by shaking quantum dots in a planar microcavity, Nat. Photon. 6, 30 (2012).

[9] M. R. Armstrong, E. J. Reed, K.-Y. Kim, J. H. Glownia, W. M. Howard, E. L. Piner, and J. C. Roberts, Observation of terahertz radiation coherently generated by acoustic waves, Nat. Phys. 5, 285 (2009).

[10] T. Dehoux and B. Audoin, Non-invasive optoacoustic probing of the density and stiffness of single biological cells, J. Appl. Phys. 112, 124702 (2012).

[11] V. E. Gusev and P. Ruello, Advances in applications of timedomain Brillouin scattering for nanoscale imaging, Appl. Phys. Rev. 5, 031101 (2018).

[12] J. Margueritat, A. Virgone-Carlotta, S. Monnier, H. DelanoëAyari, H. C. Mertani, A. Berthelot, Q. Martinet, X. Dagany, C. Rivière, J.-P. Rieu, and T. Dehoux, High-Frequency Mechanical Properties of Tumors Measured by Brillouin Light Scattering, Phys. Rev. Lett. 122, 018101 (2019).

[13] Y. Imade, R. Ulbricht, M. Tomoda, O. Matsuda, G. Seniutinas, S. Juodkazis, and O. B. Wright, Gigahertz optomechanical modulation by split-ring-resonator nanophotonic meta-atom arrays, Nano Lett. 17, 6684 (2017).

[14] R. Berte, F. Della Picca, M. Poblet, Y. Li, E. Cortés, R. V. Craster, S. A. Maier, and A. V. Bragas, Acoustic Far-Field Hypersonic Surface Wave Detection with Single Plasmonic Nanoantennas, Phys. Rev. Lett. 121, 253902 (2018). 
[15] F. Xu, Y. Guillet, S. Ravaine, and B. Audoin, All-optical in-depth detection of the acoustic wave emitted by a single gold nanorod, Phys. Rev. B 97, 165412 (2018).

[16] T. Stoll, P. Maioli, A. Crut, S. Rodal-Cedeira, I. PastorizaSantos, F. Vallée, and N. Del Fatti, Time-resolved investigations of the cooling dynamics of metal nanoparticles: Impact of environment, J. Phys. Chem. C 119, 12757 (2015).

[17] G. Rozas, M. F. Pascual Winter, B. Jusserand, A. Fainstein, B. Perrin, E. Semenova, and A. Lemaître, Lifetime of $\mathrm{THz}$ Acoustic Nanocavity Modes, Phys. Rev. Lett. 102, 015502 (2009).

[18] C. Thomsen, J. Strait, Z. Vardeny, H. J. Maris, J. Tauc, and J. J. Hauser, Coherent Phonon Generation and Detection by Picosecond Light Pulses, Phys. Rev. Lett. 53, 989 (1984).

[19] C. Thomsen, H. T. Grahn, H. J. Maris, and J. Tauc, Surface generation and detection of phonons by picosecond light pulses, Phys. Rev. B 34, 4129 (1986).

[20] A. Huynh, N. D. Lanzillotti-Kimura, B. Jusserand, B. Perrin, A. Fainstein, M. F. Pascual-Winter, E. Peronne, and A. Lemaître, Subterahertz Phonon Dynamics in Acoustic Nanocavities, Phys. Rev. Lett. 97, 115502 (2006).

[21] A. E. Bruchhausen, N. D. Lanzillotti-Kimura, B. Jusserand, A. Soukiassian, L. Xie, X. Q. Pan, T. Dekorsy, D. G. Schlom, and A. Fainstein, Acoustic confinement phenomena in oxide multifunctional nanophononic devices, Phys. Rev. Mater. 2, 106002 (2018).

[22] N. D. Lanzillotti-Kimura, A. Fainstein, C. A. Balseiro, and B. Jusserand, Phonon engineering with acoustic nanocavities: Theoretical considerations on phonon molecules, band structures, and acoustic Bloch oscillations, Phys. Rev. B 75, 024301 (2007).

[23] H. Sanchis-Alepuz, Y. A. Kosevich, and J. Sánchez-Dehesa, Acoustic Analogue of Electronic Bloch Oscillations and Resonant Zener Tunneling in Ultrasonic Superlattices, Phys. Rev. Lett. 98, 134301 (2007).

[24] A. Yariv, Y. Xu, R. K. Lee, and A. Scherer, Coupled-resonator optical waveguide: a proposal and analysis, Opt. Lett. 24, 711 (1999).

[25] N. D. Lanzillotti-Kimura, A. Fainstein, B. Perrin, B. Jusserand, O. Mauguin, L. Largeau, and A. Lemaître, Bloch Oscillations of THz Acoustic Phonons in Coupled Nanocavity Structures, Phys. Rev. Lett. 104, 197402 (2010).

[26] M. Esmann, F. R. Lamberti, A. Lemaître, and N. D. LanzillottiKimura, Topological acoustics in coupled nanocavity arrays, Phys. Rev. B 98, 161109(R) (2018).

[27] M. Esmann, F. R. Lamberti, P. Senellart, I. Favero, O. Krebs, L. Lanco, C. Gomez Carbonell, A. Lemaître, and N. D. LanzillottiKimura, Topological nanophononic states by band inversion, Phys. Rev. B 97, 155422 (2018).

[28] M. Esmann and N. D. Lanzillotti-Kimura, A topological view on optical and phononic Fabry-Perot microcavities through the Su-Schrieffer-Heeger model, Appl. Sci. 8, 527 (2018).

[29] G. Arregui, O. Ortíz, M. Esmann, C. M. Sotomayor-Torres, C. Gomez-Carbonell, O. Mauguin, B. Perrin, A. Lemaître, P. D. García, and N. D. Lanzillotti-Kimura, Coherent generation and detection of acoustic phonons in topological nanocavities, APL Photon. 4, 030805 (2019)

[30] M. Cardona, G. Güntherodt, and G. Abstreiter, Light Scattering in Solids V: Superlattices and Other Microstructures, Topics in Applied Physics (Springer, Berlin, 1989).
[31] M. Lermer, N. Gregersen, F. Dunzer, S. Reitzenstein, S. Höfling, J. Mørk, L. Worschech, M. Kamp, and A. Forchel, Bloch-Wave Engineering of Quantum Dot Micropillars for Cavity Quantum Electrodynamics Experiments, Phys. Rev. Lett. 108, 057402 (2012).

[32] G. Malpuech and A. Kavokin, Picosecond beats in coherent optical spectra of semiconductor heterostructures: Photonic Bloch and exciton-polariton oscillations, Semicond. Sci. Technol. 16, R1 (2001).

[33] V. Agarwal, J. A. del Río, G. Malpuech, M. Zamfirescu, A. Kavokin, D. Coquillat, D. Scalbert, M. Vladimirova, and B. Gil, Photon Bloch Oscillations in Porous Silicon Optical Superlattices, Phys. Rev. Lett. 92, 097401 (2004).

[34] C. Kittel, Introduction to Solid State Physics, 8th ed. (Wiley, New York, 2004).

[35] Y. Ezzahri, S. Grauby, J. M. Rampnoux, H. Michel, G. Pernot, W. Claeys, S. Dilhaire, C. Rossignol, G. Zeng, and A. Shakouri, Coherent phonons in Si/SiGe superlattices, Phys. Rev. B 75, 195309 (2007).

[36] J. Zak, Symmetry criterion for surface states in solids, Phys. Rev. B 32, 2218 (1985).

[37] M. Xiao, Z. Q. Zhang, and C. T. Chan, Surface Impedance and Bulk Band Geometric Phases in One-Dimensional Systems, Phys. Rev. X 4, 021017 (2014).

[38] G. Crosnier, D. Sanchez, A. Bazin, P. Monnier, S. Bouchoule, R. Braive, G. Beaudoin, I. Sagnes, R. Raj, and F. Raineri, High $\mathrm{Q}$ factor InP photonic crystal nanobeam cavities on silicon wire waveguides, Opt. Lett. 41, 579 (2016).

[39] S. Combrié, G. Lehoucq, G. Moille, A. Martin, and A. De Rossi, Comb of high-Q resonances in a compact photonic cavity, Laser Photon. Rev. 11, 1700099 (2017).

[40] M. F. Pascual Winter, G. Rozas, A. Fainstein, B. Jusserand, B. Perrin, A. Huynh, P. O. Vaccaro, and S. Saravanan, Selective Optical Generation of Coherent Acoustic Nanocavity Modes, Phys. Rev. Lett. 98, 265501 (2007).

[41] M. Bayer, T. Gutbrod, J. P. Reithmaier, A. Forchel, T. L. Reinecke, P. A. Knipp, A. A. Dremin, and V. D. Kulakovskii, Optical Modes in Photonic Molecules, Phys. Rev. Lett. 81, 2582 (1998).

[42] A. Dousse, J. Suffczynski, A. Beveratos, O. Krebs, A. Lemaître, I. Sagnes, J. Bloch, P. Voisin, and P. Senellart, Ultrabright source of entangled photon pairs, Nature (London) 466, 217 (2010).

[43] M. Zhang, C. Wang, Y. Hu, A. Shams-Ansari, T. Ren, S. Fan, and M. Loncar, Electronically programmable photonic molecule, Nat. Photon. 13, 36 (2019).

[44] E. Péronne, N. Chuecos, L. Thevenard, and B. Perrin, Acoustic solitons: A robust tool to investigate the generation and detection of ultrafast acoustic waves, Phys. Rev. B 95, 064306 (2017).

[45] T. C. Zhu, H. J. Maris, and J. Tauc, Attenuation of longitudinalacoustic phonons in amorphous $\mathrm{SiO}_{2}$ at frequencies up to 440 GHz, Phys. Rev. B 44, 4281 (1991).

[46] T.-M. Liu, S.-Z. Sun, C.-F. Chang, C.-C. Pan, G.-T. Chen, J.-I. Chyi, V. Gusev, and C.-K. Sun, Anharmonic decay of subterahertz coherent acoustic phonons in GaN, Appl. Phys. Lett. 90, 041902 (2007).

[47] R. Legrand, A. Huynh, B. Jusserand, B. Perrin, and A. Lemaître, Direct measurement of coherent subterahertz acoustic phonons mean free path in GaAs, Phys. Rev. B 93, 184304 (2016). 
[48] J. Lamb, J. Richter, and H. E. M. Barlow, Anisotropic acoustic attenuation with new measurements for quartz at room temperatures, Proc. R. Soc. London Ser. A. 293, 479 (1966).

[49] M. J. Frazier and M. I. Hussein, Generalized Bloch's theorem for viscous metamaterials: Dispersion and effective properties based on frequencies and wavenumbers that are simultaneously complex, C. R. Phys. 17, 565 (2016).

[50] B. G. Helme and P. J. King, The phonon viscosity tensor of Si, Ge, GaAs, and InSb, Phys. Status Solidi (a) 45, K33 (1978).

[51] R. L. Hall, W. Lucha, and F. F. Schoberl, Energy bounds for the spinless Salpeter equation: Harmonic oscillator, J. Phys. A: Math. Gen. 34, 5059 (2001).

[52] N. D. Lanzillotti-Kimura, A. Fainstein, A. Lemaître, and B. Jusserand, Nanowave devices for terahertz acoustic phonons, Appl. Phys. Lett. 88, 083113 (2006).

[53] A. Fainstein, N. D. Lanzillotti-Kimura, B. Jusserand, and B. Perrin, Strong Optical-Mechanical Coupling in a Vertical GaAs/AlAs Microcavity for Subterahertz Phonons and NearInfrared Light, Phys. Rev. Lett. 110, 037403 (2013).

[54] M. De Luca, C. Fasolato, M. A. Verheijen, Y. Ren, M. Y. Swinkels, S. Kölling, E. P. A. M. Bakkers, R. Rurali, X. Cartoixà, and I. Zardo, Phonon engineering in twinning superlattice nanowires, Nano Lett. 19, 4702 (2019).

[55] G. Arregui, N. D. Lanzillotti-Kimura, C. M. Sotomayor-Torres, and P. D. García, Anderson Photon-Phonon Colocalization in
Certain Random Superlattices, Phys. Rev. Lett. 122, 043903 (2019).

[56] S. Anguiano, A. E. Bruchhausen, B. Jusserand, I. Favero, F. R. Lamberti, L. Lanco, I. Sagnes, A. Lemaître, N. D. Lanzillotti-Kimura, P. Senellart, and A. Fainstein, Micropillar Resonators for Optomechanics in the Extremely High 19-95-GHz Frequency Range, Phys. Rev. Lett. 118, 263901 (2017).

[57] S. Anguiano, P. Sesin, A. E. Bruchhausen, F. R. Lamberti, I. Favero, M. Esmann, I. Sagnes, A. Lemaître, N. D. LanzillottiKimura, P. Senellart, and A. Fainstein, Scaling rules in optomechanical semiconductor micropillars, Phys. Rev. A 98, 063810 (2018).

[58] F. R. Lamberti, Q. Yao, L. Lanco, D. T. Nguyen, M. Esmann, A. Fainstein, P. Sesin, S. Anguiano, V. Villafañe, A. Bruchhausen, P. Senellart, I. Favero, and N. D. Lanzillotti-Kimura, Optomechanical properties of GaAs/AlAs micropillar resonators operating in the $18 \mathrm{GHz}$ range, Opt. Express 25, 24437 (2017).

[59] M. Xiao, G. Ma, Z. Yang, P. Sheng, Z. Q. Zhang, and C. T. Chan, Geometric phase and band inversion in periodic acoustic systems, Nat. Phys. 11, 240 (2015).

[60] N. D. Lanzillotti-Kimura, A. Fainstein, B. Jusserand, and A. Lemaître, Resonant Raman scattering of nanocavity-confined acoustic phonons, Phys. Rev. B 79, 035404 (2009). 